\title{
Transportation Time is Significantly Decreased in Acute Ischemic Stroke Patients Under Drip-and-Ship Paradigm for Thrombolysis
}

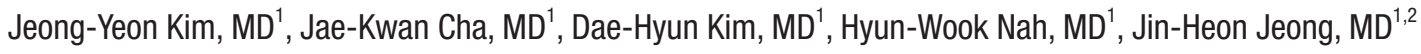 \\ ${ }^{1}$ Department of Neurology, Stroke Center, Dong-A University Hospital, Dong-A University College of Medicine, Busan; ${ }^{2}$ Department of Intensive Care Medicine, Dong-A \\ University Hospital, Dong-A University College of Medicine, Busan, Korea
}

Background: A delay of transfer for patients with acute stroke needing emergent revascularization is a huge hurdle for efficacy of revascularization. The objective of this study was to investigate changes of transportation time calculated by image to door (ITD) time (from checking brain images at first contact hospital to arriving at our emergency center) before and after 2015.

Methods: This study was performed in a retrospective manner from 2013 into 2017. Acute ischemic stroke (AIS) patients having intravenous thrombolysis and/or mechanical thrombectomy during the observation period were enrolled. Among them, those who had revascularization under 'Drip-and-Ship' or 'Ship-and-Drip' paradigm were selected

Results: During the observation period, 225 patients were treated under 'Drip-and-Ship' or 'Ship-and-Drip' paradigm. Twenty-three were excluded due the lack of detailed data. Among 202 patients, 73 and 129 were treated under Drip-and-Ship and Ship-and-Drip paradigms, respectively. In 2013, 35 patients from 18 hospitals (median distance, $25 \mathrm{~km}$ ) were transferred to our regional stroke center and their median ITD time was 116 minutes. It was gradually decreased after 2015. In 2017, ITD time was significantly $(P<0.01)$ shortened to 85 minutes without significant changes in transfer distance. The median onset to puncture time was also significantly $(P=0.03)$ decreased from 365 minutes in 2013 to 270 minutes in 2017.

Conclusion: Our results implicate that many hospitals in our stroke region might have recognized the importance of rapid transportation for AIS after 2015

J Neurocrit Care 2018;11(2):86-92

Key words: Stroke; Thrombectomy; Transportation

\author{
Received October 3, 2018 \\ Revised October 19, 2018 \\ Accepted November 11, 2018 \\ Corresponding Author: \\ Jin-Heon Jeong, MD \\ Departments of Intensive Care \\ Medicine and Neurology, Stroke Center, \\ Dong-A University Hospital, Dong-A \\ University College of Medicine, 26 \\ Daesingongwon-ro, Seo-gu, Busan \\ 49201, Korea \\ Tel: +82-51-240-5266 \\ Fax: +82-51-244-8338 \\ E-mail: jhjeong@dau.ac.kr
}

Copyright $\odot 2018$ The Korean Neurocritical Care Society

\section{INTRODUCTION}

After five successful clinical trials for mechanical thrombectomy have been published in 2015, endovascular treat- ment for acute ischemic stroke (AIS) caused by acute largevessel occlusion (ALVO) in anterior circulation is now the standard of care for patients presenting within 6 hours of symptom onset. ${ }^{1-5}$ However, this treatment option cannot

cc This is an Open Access article distributed under the terms of the Creative Commons Attribution Non-Commercial License (http://creativecommons.org/licenses/by$\mathrm{nc} / 4.0$ ) which permits unrestricted non-commercial use, distribution, and reproduction in any medium, provided the original work is properly cited. 
be generalized like using intravenous (IV) tissue plasminogen activator (t-PA) in AIS. Many previous data have shown that roughly fewer than one-third of stroke centers are comprehensive stroke centers with on-site neurointerventionist. ${ }^{6}$ Therefore, not all AIS patients with ALVO can be directly admitted to a comprehensive stroke center that can offer both IV thrombolysis and mechanical thrombectomy. To solve this problem, Drip-and-Ship (D-S) model using IV t-PA at the nearest hospital before being transferred into a comprehensive stroke center for mechanical thrombectomy has been proposed for AIS patients.

The critical problem of the D-S model is a longer process time by a time delay of inter-hospital transportation..$^{7-10}$ However, several reports after 2015 have shown a decrement of inter-hospital transportation due to better recognition of the importance of the process time after the publication of trials and a learning curve effect at several levels (such as advanced prehospital notification, cooperation between centers, and rapid response of neurointerventionist). ${ }^{10,11}$ The objective of this study was to investigate changes of inter-hospital transportation time calculated as image to door (ITD) time (from first image at primary hospital to arrival time at emergency room (ER) of a comprehensive stroke center) before and after 2015 in Busan metropolitan area, Korea.

\section{METHODS}

This was a retrospective observational study. It was approved by Dong-A University Hospital (DAUH) Institutional Review Board. For the purpose of this study, AIS patients with thrombolysis (IV t-PA and/or mechanical thrombectomy) under D-S or Ship-and-Drip (S-D) paradigm between January 2013 and December 2017 were identified from our stroke registry. DAUH is a tertiary teaching hospital located in the Busan metropolitan area (size, 765.64 km² population, 4 million). Our stroke center is a regional comprehensive stroke center in the Busan metropolitan area supported by the Korean government. When the comprehensive stroke center was opened, an 8-bed stroke care unit was formed for the care of acute stroke patients. This unit contains a telemetry system for monitoring of blood pressure, pulse, respiration, and oxygenation. A multidisciplinary group developed stroke care pathways to guide the evaluation and treatment of each stroke subtype. Stroke treatment was generally administered according to a wellorganized team approach, e.g., IV thrombolysis, endovascular treatment, hemicraniectomy, and carotid endarterectomy.

Our strategy for using IV t-PA is based on magnetic resonance imaging (MRI) in principle. Our door to image time is maintained within 23 minutes. In 2015, a hotline system (1899-0215 in conjunction with the Korean Emergency Medical Information System [KEMS; 119]) was implemented in our hospital for prehospital notification. The primary role of the KEMS is to connect a patient needing an emergency or operation in a community hospital with appropriate available hospital. ${ }^{12}$

\section{Critical pathway for hyperacute ischemic stroke}

Our stroke critical pathway is based on CODE RED program, ${ }^{13}$ a kind of computerized physician order entry system that enables activation, communication, notification, entering predetermined standing order sets, and providing protocols and guidelines. ${ }^{11}$ In our stroke code system, MRI is routinely performed to screen patients for thrombolytic therapy. However, when an immediate MRI is unavailable because it is being used for other patients, brain computed tomography (CT) is used for IV t-PA. It is administered according to the National Institute of Neurological Disorders and Stroke criteria. ${ }^{12}$ For such cases, MRI was performed before mechanical thrombectomy.

\section{Definition of time points}

In this study, two time points were used to evaluate time intervals of D-S paradigm for thrombolysis as follows: ITD time and onset to puncture (OTP) time (from symptom onset to groin puncture time).

\section{Statistical analysis}

In this study, data of National Institute of Health Stroke 
Scale (NIHSS), ITD time, OTP time, and transfer distance for included patients from 2013 into 2017 were collected. These parameters are expressed as median (interquartile range [IQR]). Statistical analysis of the serial trend of above parameters compared to those in 2013 and 2014 were conducted by using Kruskal-Wallis test. A $P$ value of less than 0.05 was considered statistically significant. All statistical analyses were carried out using the SPSS version 21.0 (IBM Corp., Armonk, NY, USA).

\section{RESULTS}

During the observation period, 806 patients received IV t-PA and/or mechanical thrombectomy. Among them, 225 subjects (27.9\%) were treated under D-S or S-D paradigm. Of these 225 patients, 23 were excluded because of the lack of detailed data. Finally, 202 patients were enrolled in this study. Of these 202 patients, 73 were treated under D-S while 129 were treated under S-D. A total of 134 patients received IV t-PA only. Seventeen received mechanical thrombectomy only while 51 received mechanical thrombectomy after receiving IV t-PA. The median image to arrival time was 116 minutes (IQR, 92) in 2013. After 2015, the time interval gradually decreased. It was significantly $(P<0.01)$ decreased to 85 minutes $(I Q R, 67)$ in 2017. The initial median neurologic severity measured by NIHSS was 9 (IQR, 10) in 2013. There was no difference in NIHSS during the observation period (Table 1, Fig. 1).

Regarding characteristics of hospitals from which acute stroke patients were transferred to our center, more than $95 \%$ of hospitals having their own stroke neurologist or neurosurgeon and Angio CT were capable of treating acute stroke. The median transfer distance was $25 \mathrm{~km}(\mathrm{IQR}, 24)$ in 2013. It showed no significant difference during the observation period (Fig. 2).

Among 202 patients enrolled for this study, a total of 68 received mechanical thrombectomy during the observation period. In 2013, the median OTP time was 365 minutes (IQR, 97.5). It was significantly decreased to 270 minutes (IQR, 65) in 2015, 285 minutes (IQR, 189) in 2016, and 270 minutes $(I Q R, 72)$ in $2017(P=0.03$, Table 1, Fig. 1).

Table 1. Changes of clinical, geographic, and time parameters during the observation period (from 2013 to 2017)

\begin{tabular}{|c|c|c|c|c|c|c|}
\hline & 2013 & 2014 & 2015 & 2016 & 2017 & $P$ value \\
\hline \multicolumn{7}{|c|}{ NIHSS (n=202) } \\
\hline Median & 9 & 14 & 12 & 9 & 13 & $0.55^{*}$ \\
\hline $\mathrm{IQR}$ & 10 & 10.75 & 14 & 9.75 & 12 & $0.56^{\dagger}$ \\
\hline Q1, Q3 & 5,15 & $6.5,17.25$ & 4,18 & $6,15.75$ & 5,17 & \\
\hline \multicolumn{7}{|c|}{ ITD time $(n=202)$} \\
\hline Median & 116 & 124 & 111.5 & 87 & 85 & $<0.01 *$ \\
\hline IQR & 92 & 77.75 & 81.25 & 36.5 & 67 & $<0.01^{\dagger}$ \\
\hline Q1, Q3 & 65,157 & $84.75,162.5$ & $77.25,158.5$ & $67.25,103.75$ & 56,123 & \\
\hline \multicolumn{7}{|c|}{ OTP time $(n=70)$} \\
\hline Median & 365 & 378 & 270 & 285 & 270 & $0.03 *$ \\
\hline IQR & 97.5 & 196 & 65 & 189 & 72 & $0.05^{\dagger}$ \\
\hline Q1, Q3 & $287.5,385$ & $287.75,483.75$ & 230,295 & $216.5,405.5$ & 223,295 & \\
\hline \multicolumn{7}{|c|}{ Distance $(n=202)$} \\
\hline Median & 25 & 26 & 23.5 & 25 & 23 & $0.53 *$ \\
\hline IQR & 24 & 35.5 & 40 & 37.25 & 36 & $0.68^{\dagger}$ \\
\hline Q1, Q3 & 17,41 & $17.75,53.25$ & 12,52 & $14,51.25$ & 14,50 & \\
\hline
\end{tabular}

NIHSS, National Institute of Health Stroke Scale; IQR, interquartile range; ITD, image to door; OTP, onset to puncture.

*Changes of parameters compared to 2013.

${ }^{\dagger}$ Changes of parameters compared to 2014. 
A

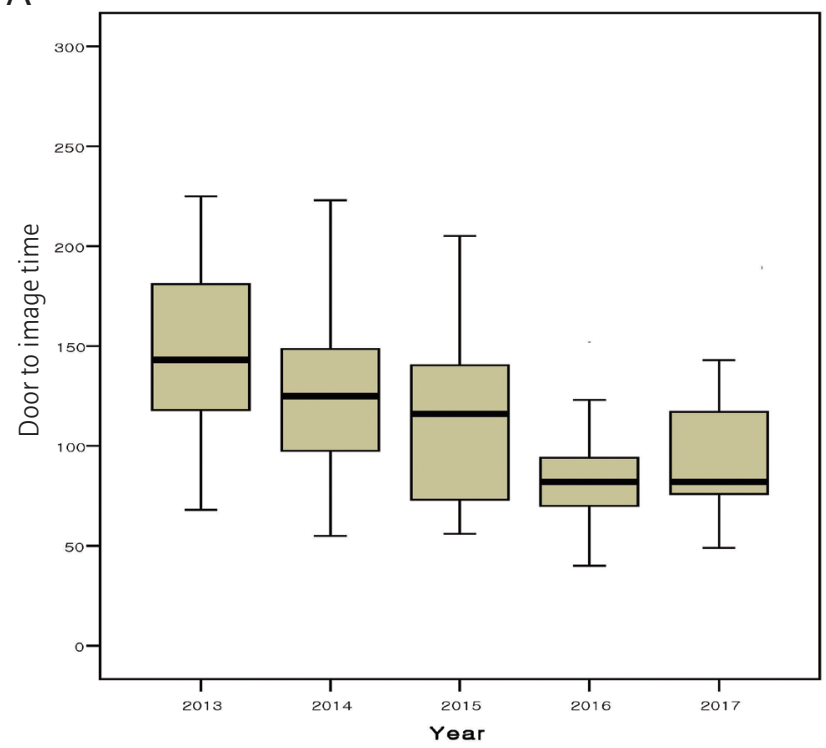

B

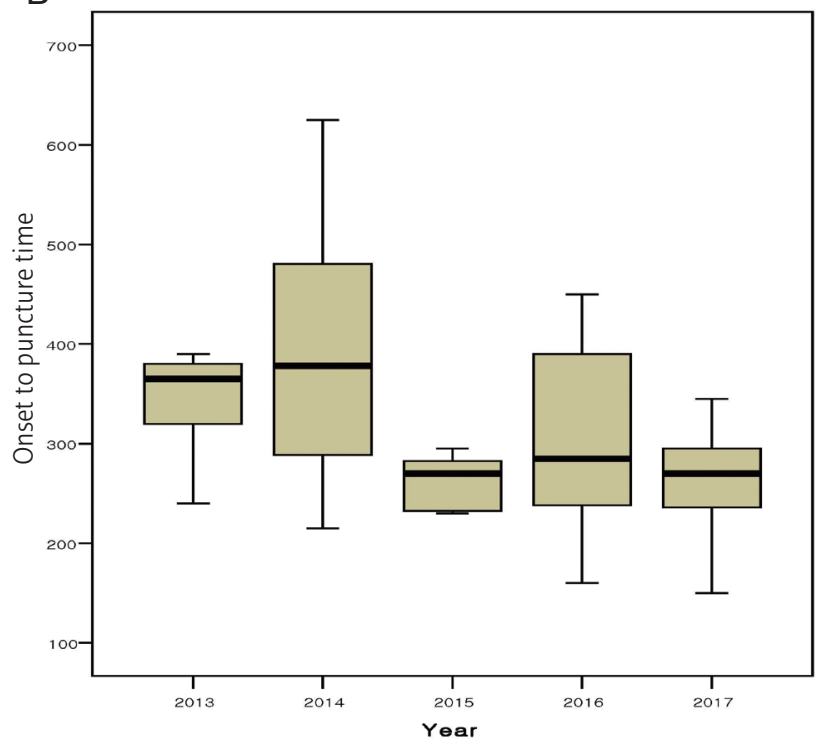

Figure 1. Box plots for door to image time (A) and onset to puncture time (B). The horizontal line inside each box indicates the median. Top and bottom of the box indicate interquartile range while I bars indicate 5 th and 95 th percentiles.

A

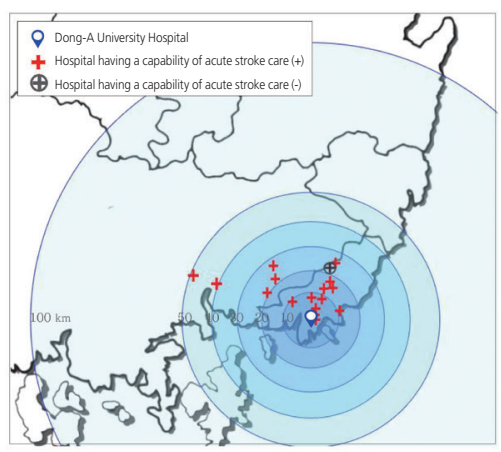

D

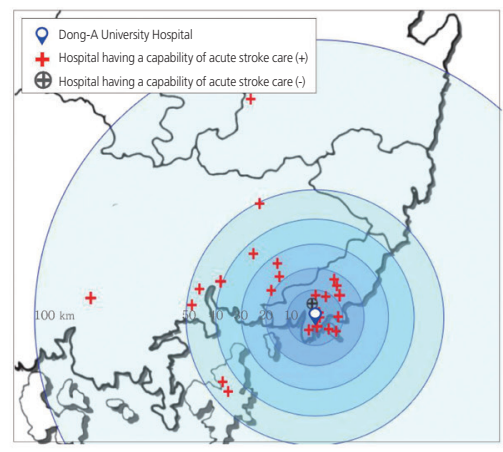

$\mathrm{B}$

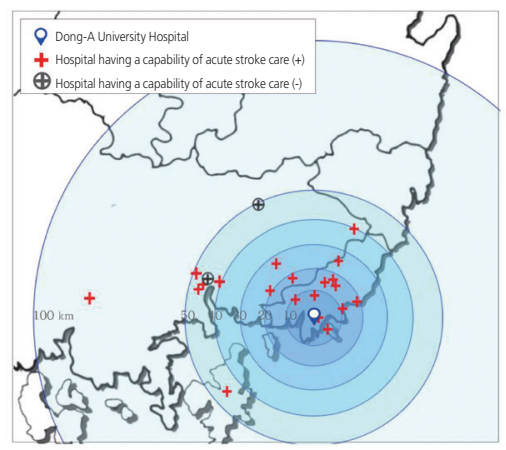

E

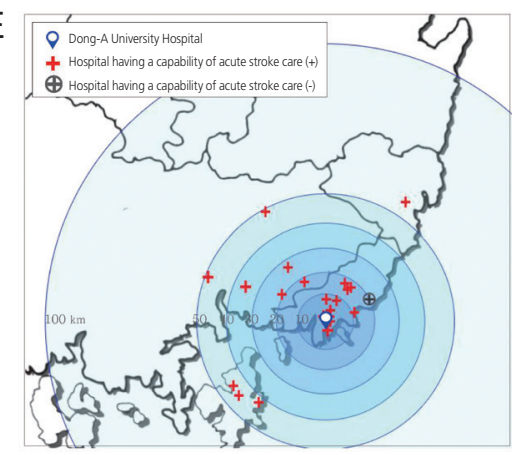

C

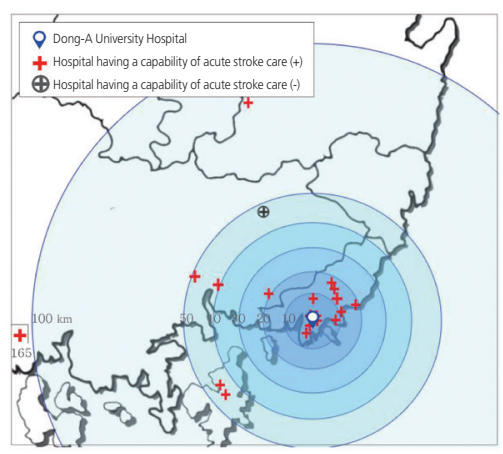

Figure 2. Location of Dong-A University Stroke Center and distribution of transfer hospitals. (A) In 2013, 18 neighboring hospitals that transferred patients to our center. (B) In 2014, 23 neighboring hospitals that transferred patients to our center. (C) In 2015,19 neighboring hospitals that transferred patients to our center. (D) In 2016, 27 neighboring hospitals that transferred patients to our center. (E) In 2017, 24 neighboring hospitals that transferred patients to our center. 
After 90 days of thrombolysis, the rate of poor outcome (defined as modified Rankin scores of 3-6) was $54.3 \%$ in $2013,66.7 \%$ in $2014,52.9 \%$ in $2015,57.7 \%$ in 2016 , and $51.3 \%$ in 2017 .

\section{DISCUSSION}

It is well-known that inter-hospital transfer time is the most important factor associated with treatment delay for mechanical thrombectomy. ${ }^{7-10}$ Therefore, decreasing the time delay might be essential to achieve efficacious D-S paradigm for mechanical thrombectomy in our regional boundary of stroke care. ${ }^{14-18}$

Transfer time from the first call to arriving at an ER of a hospital for Busan metropolitan area is about $25 \mathrm{~min}$ utes. ${ }^{19}$ Such a short time from home to a neighboring ER makes our emergency medical service (EMS) paramedics unable to detect the presence of ALVO on EMS ambulance. Due to such problem, it is impossible to use the mothership paradigm for mechanical thrombectomy in Busan metropolitan area.

Busan metropolitan area has a unique pattern of D-S paradigm, showing more severe neurologic deficits at admission and much worse outcome after thrombolysis. ${ }^{12}$ This result could be explained that many hospitals in Busan have their own stroke neurologist and their own Angio CT and/or MRI. Therefore, in our metropolitan area, almost all patients who received IV t-PA were initially treated by a stroke neurologist or neurosurgeon in a community hospital while only selected patients were transferred to our comprehensive stroke center due to the lack of mechanical thrombectomy or more intensive care after a phone consultation. In line with our previous data, ${ }^{12}$ the present study showed that more than 95\% hospitals that sent patients to our center had capability for treating acute stroke. This pattern was similarly found in CRCS-K registry data in Korea showing that only patients with severe neurological deficits requiring further intensive care after t-PA administration were likely to be transferred to comprehensive stroke centers. 20
The inter-hospital transfer time calculated by ITD time was about 116 minutes in 2013 in the present study. This time interval was significantly delayed compared to previous reports in other countries showing 85 minutes of interhospital transfer time from primary stroke center to comprehensive stroke center. ${ }^{21,22}$ Several factors might have limited rapid transfer of acute stroke patients between hospitals. First, there has been no governance to control transportation of stroke patients in Busan metropolitan area. Second, after deciding to transfer ALVO patients to a comprehensive stroke center, a substantial amount of time is needed for administrative procedures, release of medical records including hard copies of CT or MRI images, and checkout procedures at discharge. In fact, almost all time delays of inter-hospital transfer were caused by work-up procedure in a primary hospital. ${ }^{23,24}$ Third, it was not easy for neighboring community hospitals to contact stroke physicians in our comprehensive stroke center.

In our stroke boundary, D-S or S-D paradigm for patients undergoing thrombolysis has already been voluntarily performed by neighboring hospitals and our comprehensive stroke center from 2009. 12,19,25 To make ensure a prenotification between neighboring hospitals and our comprehensive stroke center, we have opened our individual mobile phones to neighboring hospitals in our stroke region. Since 2015, we have used the hotline number that is connected to neighboring hospitals and EMS paramedics in Busan metropolitan area. ${ }^{25}$ As a consequence of above efforts, the proportion of D-S or S-D paradigm was $27.9 \%$ during the observation period in our center. This proportion is twice higher than those in other similar comprehensive stroke centers in CRCS- $\mathrm{K}$ registry. ${ }^{20}$ Particularly, there were no significant differences in transportation time of stroke patients to our comprehensive stroke center from participating hospitals between our previous 2009 study ${ }^{12}$ and the present data. Sun and colleagues ${ }^{26}$ have found that the more often patients are transferred by the same hospital to a comprehensive stroke center, the more often transfer time interval is decreased. The significant decrement of inter-hospital transfer time in this study might be due to combined results of efficient pre-hospital notification by 
using our hot line system and increased recognition of rapid transportation for acute stroke patients with a learning curve on how to effectively transfer AIS patients into comprehensive stroke center after 2015 with five successful mechanical thrombectomy trials.

The median OTP time was also significantly decreased from 365 minutes in 2013 to 270 minutes in 2017 in the present study. This beneficial effect was mainly due to a significant reduction in inter-hospital transfer time. Prehospital notification by a stroke physician in neighboring community hospitals might have decreased intra-hospital processing time for mechanical thrombectomy in our comprehensive stroke center. In MR CLEAN trial, ${ }^{23}$ an increased time to arrival at the ER in transferred patients was clearly associated with a decrease in the following time to groin puncture. This paradoxical effect is likely caused by the fact that a comprehensive stroke center is pre-notified before arrival at the ER, making it possible to reduce the door to puncture time. However, we did not show this beneficial effect by a pre-hospital notification on D-S paradigm in detail.

Like several regions such as Paris, ${ }^{10}$ Frankfurt, ${ }^{11}$ and several cities in Holland ${ }^{23}$ having geographic proximity that achieved effective organized stroke network based on D-S paradigm, this strategy might be applied in Busan for mechanical thrombectomy. Reducing transportation time to a comprehensive stroke center might be essential for successful D-S paradigm. In this sense, significant shortening of ITD time could be an important cornerstone to build up an effective organized stroke center in our stroke boundary. Although ITD time was significantly decreased to 85 minutes in 2017, it was more than 30 minutes longer than that of previous reports ${ }^{10,11,23}$ with organized stroke network in several European countries. This difference might be associated with different characteristics of medical systems. Unlike European countries, more than $70 \%$ of hospitals in Korea belong to the private sector. This kind of difference might have influenced the delay of inter-hospital transfer time in Busan metropolitan area. More detailed investigation is needed to test this hypothesis in the future.

This study has several limitations. First, this was a retro- spective study. Second, we were not able to obtain the door to needle time in patients treated under the D-S paradigm.

\section{CONCLUSION}

The inter-hospital transfer time was significantly decreased after 2015 in our stroke boundary of Busan metropolitan area. Our results implicated that many hospitals in our stroke region might have recognized the importance of rapid transportation for acute ischemic stroke after 2015. Based on this achievement, we should be able to build up an organized stroke system having a form of D-S paradigm in the future.

\section{REFERENCES}

1. Berkhemer OA, Fransen PS, Beumer D, van den Berg LA, Lingsma HF, Yoo AJ, et al. A randomized trial of intraarterial treatment for acute ischemic stroke. $N$ Engl / Med 2015;372:11-20

2. Goyal M, Demchuk AM, Menon BK, Eesa M, Rempel JL, Thornton J, et al. Randomized assessment of rapid endovascular treatment of ischemic stroke. $N$ Engl / Med 2015;372:1019-30.

3. Campbell BC, Mitchell PJ, Kleinig TJ, Dewey HM, Churilov L, Yassi N, et al. Endovascular therapy for ischemic stroke with perfusion-imaging selection. N Engl / Med 2015;372:100918

4. Saver JL, Goyal M, Bonafe A, Diener HC, Levy El, Pereira VM, et al. Stent-retriever thrombectomy after intravenous t-PA vs. t-PA alone in stroke. N Engl / Med 2015;372:2285-95.

5. Jovin TG, Chamorro A, Cobo E, de Miquel MA, Molina CA, Rovira A, et al. Thrombectomy within 8 hours after symptom onset in ischemic stroke. N Eng/ / Med 2015;372:2296-306.

6. Adeoye O, Albright KC, Carr BG, Wolff C, Mullen MT, Abruzzo $T$, et al. Geographic access to acute stroke care in the United States. Stroke 2014;45:3019-24.

7. Saver JL, Goyal M, van der Lugt A, Menon BK, Majoie CB, Dippel DW, et al. Time to treatment with endovascular thrombectomy and outcomes from ischemic stroke: a metaanalysis. JAMA 2016;316:1279-88. 
8. Badhiwala JH, Nassiri F, Alhazzani W, Selim MH, Farrokhyar F, Spears J, et al. Endovascular thrombectomy for acute ischemic stroke: a meta-analysis. JAMA2015;314:1832-43.

9. Menon BK, Sajobi TT, Zhang Y, Rempel JL, Shuaib A, Thornton J, et al. Analysis of workflow and time to treatment on thrombectomy outcome in the Endovascular Treatment for Small Core and Proximal Occlusion Ischemic Stroke (ESCAPE) randomized, controlled trial. Circulation 2016;133:2279-86.

10. Gerschenfeld G, Muresan IP, Blanc R, Obadia M, Abrivard M, Piotin $\mathrm{M}$, et al. Two paradigms for endovascular thrombectomy after intravenous thrombolysis for acute ischemic stroke. JAMA Neurol 2017;74:549-56.

11. Daubail B, Ricolfi F, Thouant P, Vogue C, Chavent A, Osseby $\mathrm{GV}$, et al. Impact of mechanical thrombectomy on the organization of the management of acute ischemic stroke. Eur Neurol 2016;75:41-7.

12. Cha JK, Nah HW, Kang MJ, Kim DH, Park HS, Kim SB, et al. Outcomes after tissue plasminogen activator administration under the drip and ship paradigm may differ according to the regional stroke care system. / Stroke Cerebrovasc Dis 2014;23:160-3.

13. Sohn SW, Park HS, Cha JK, Nah HW, Kim DH, Kang MJ, et al. A systemized stroke code significantly reduced time intervals for using intravenous tissue plasminogen activator under magnetic resonance imaging screening. / Stroke Cerebrovasc Dis 2015:24:465-72.

14. Asif KS, Lazzaro MA, Zaidat O. Identifying delays to mechanical thrombectomy for acute stroke: onset to door and door to clot times. / Neurointerv Surg 2014;6:505-10.

15. Goyal M, Almekhlafi MA, Fan L, Menon BK, Demchuk AM, Yeatts SD, et al. Evaluation of interval times from onset to reperfusion in patients undergoing endovascular therapy in the interventional management of stroke III trial. Circulation 2014; 130:265-72

16. Sheth KN, Smith EE, Grau-Sepulveda MV, Kleindorfer D, Fonarow GC, Schwamm LH. Drip and ship thrombolytic therapy for acute ischemic stroke: use, temporal trends, and outcomes. Stroke 2015;46:732-9.

17. Menon BK, Almekhlafi MA, Pereira VM, Gralla J, Bonafe A, Davalos A, et al. Optimal workflow and process-based performance measures for endovascular therapy in acute ischemic stroke: analysis of the Solitaire FR thrombectomy for acute revascularization study. Stroke 2014;45:2024-9.

18. Goyal M, Jadhav AP, Bonafe A, Diener H, Mendes Pereira V, Levy E, et al. Analysis of workflow and time to treatment and the effects on outcome in endovascular treatment of acute ischemic stroke: results from the SWIFT PRIME randomized controlled trial. Radiology 2016;279:888-97.

19. Kim HJ, Kim DH, Park HI, Lee IY, Cha JK. Prehospital delay factors after stroke and paramedic identification of stroke patients in a metropolitan city emergency medical service system. J Korean Neurol Assoc 2011;29:89-94.

20. Park MS, Lee JS, Park TH, Cho YJ, Hong KS, Park JM, et al. Characteristics of the drip-and-ship paradigm for patients with acute ischemic stroke in South Korea. / Stroke Cerebrovasc Dis 2016;25:2678-87.

21. Kodankandath TV, Shaji J, Kohn N, Arora R, Salamon E, Libman RB, et al. Poor hypertension control and longer transport times are associated with worse outcome in drip-andship stroke patients. / Stroke Cerebrovasc Dis 2016;25:188790.

22. Asaithambi G, Chaudhry SA, Hassan AE, Rodriguez GJ, Suri MF, Qureshi Al. Adherence to guidelines by emergency medical services during transport of stroke patients receiving intravenous thrombolytic infusion. / Stroke Cerebrovasc Dis 2013:22:e42-5

23. Venema E, Boodt N, Berkhemer OA, Rood PPM, van Zwam WH, van Oostenbrugge RJ, et al. Workflow and factors associated with delay in the delivery of intra-arterial treatment for acute ischemic stroke in the MR CLEAN trial. / Neurointerv Surg 2018;10:424-8

24. Bae HJ, Kim DH, Yoo NT, Choi JH, Huh JT, Cha JK, et al. Prehospital notification from the emergency medical service reduces the transfer and intra-hospital processing times for acute stroke patients. / Clin Neurol 2010;6:138-42.

25. Kim DH, Nah HW, Park HS, Choi JH, Kang MJ, Huh JT, et al. Impact of prehospital intervention on delay time to thrombolytic therapy in a stroke center with a systemized stroke code program. / Stroke Cerebrovasc Dis 2016;25:1665-70.

26. Sun CH, Nogueira RG, Glenn BA, Connelly K, Zimmermann $S$, Anda K, et al. "Picture to puncture": a novel time metric to enhance outcomes in patients transferred for endovascular reperfusion in acute ischemic stroke. Circulation 2013; 127:1139-48. 